\title{
Intensive Geographien - die Versorgung von extremen Frühgeburten in Deutschland aus praxistheoretischer Perspektive
}

\author{
Matthias Lahr-Kurten \\ independent researcher \\ Correspondence: Matthias Lahr-Kurten (matthias.lahr-kurten@gmx.de)
}

Received: 22 May 2016 - Revised: 24 June 2018 - Accepted: 27 June 2018 - Published: 14 November 2018

Kurzfassung. In Germany each year, more than 65000 children are born prematurely as ,preterm infants' (or ,preemies'). Some 4500 of these are born extremely premature. Despite being sites of enormous human suffering und huge financial costs, the places of treatment of these babies are almost non-existent in societal discourse. Therefore, the aim of this article is to understand this place, the so-called ,NICUs ' - Neonatal Intensive Care Units, places that seem isolated from the rest of society, but are tightly connected to it.

In order to reach this aim, a ,dense description ' of a single NICU in Germany will be given, based on a stay of 5 months by the author. The underlying perspective of that dense description is the practice theoretical approach of social philosopher Theodore Schatzki.

The description of the NICU shows that corporeality, materiality, and not least emotionality are important aspects of social phenomena. As will be seen, the underlying practice theory is a relational ontology that resists the urge to draw on micro-macro-dichotomies to understand these phenomena.

\section{Einleitung}

„Draußen wird Sie keiner verstehen“. So oder so ähnlich kann die Begrüßung lauten, wenn ein Vater/eine Mutter auf einer Frühgeborenen-Intensivstation ankommt, in der sein/ihr Kind nach der Geburt versorgt wird. Das Zitat einer Krankenschwester ${ }^{1}$ legt nahe, dass diese Orte sich derart grundlegend von anderen Orten der Gesellschaft unterscheiden, dass „draußen“ kein Verständnis für die Geschehnisse an diesem Ort erwartet werden sollte. ${ }^{2}$ FrühgeborenenIntensivstationen sind im gesellschaftlichen Diskurs nahezu

\footnotetext{
${ }^{1}$ Aus Gründen der Anonymität und der besseren Lesbarkeit wird für pflegendes, technisches und ärztliches Personal ausschließlich die weibliche Form genutzt; dies entspricht zumindest beim pflegenden Personal auch der deutlichen Mehrzahl der Beschäftigten.

${ }^{2}$ So erging es auch mir als Vater eines extrem frühgeborenen Kindes: Die Geschehnisse und der Aufbau einer FrühgeborenenIntensivstation - ihre Praktiken und Arrangements - waren mir zuvor völlig fremd. Der Versuch, diese Welt durch einen wissenschaftlichen Blick zu ,rationalisieren“ und dann auch anderen verstehbar zu machen, war die wesentliche Motivation für diesen Aufsatz.
}

nicht existent - sie werden lediglich bisweilen in dramatisierenden Reportagen über individuelle Schicksale oder tagesaktuellen Berichten zu einzelnen Fällen mangelnder Hygiene thematisiert; gegenwärtig aufkommende „Selbsthilfegruppen" in den sozialen Medien sind geschlossene Kreise der Betroffenen.

Die seit den 1960er-Jahren existierenden FrühgeborenenIntensivstationen sind jedoch in vielerlei Hinsicht gesellschaftlich relevante Orte: Weltweit kommt jedes 9. Kind vor dem Ende der 37. Schwangerschaftswoche (SSW) und damit als Frühgeburt zur Welt, während die Frühgeburtenrate in den meisten Ländern steigt (Blencowe et al., 2013:1). Innerhalb der Kategorie der Frühgeborenen existieren erhebliche Unterschiede bezüglich der Entwicklungsstadien und damit der Bedürfnisse, sodass eine Differenzierung erfolgt: Vor allem die ,extremen“ Frühgeburten, die vor Ende der 28. SSW geboren werden und zumeist unter $1000 \mathrm{~g}$ wiegen, stellen die auf Frühgeborenen-Intensivstationen versorgten Patienten dar. In Deutschland kamen 2016 über 66000 Frühgeborene zur Welt, davon über 4500 vor der 28. SSW (Bäurle, 2017). Neben „dem großen menschlichen Leid“, das sich 
hinter der beträchtlichen Zahl an Frühgeburten verbirgt, verursachen sie ,hohe Kosten in der medizinischen Versorgung": Für Deutschland wurden im Jahr 2009 die Kosten auf jährlich mindestens 735 Mio. Euro geschätzt (Kirschner et al., 2009:41; 50).

Ziel dieses Artikels wird daher sein, diese gesellschaftlich relevanten, aber zumeist unbekannten Orte aus einer geographischen Perspektive derart zu beschreiben, dass sie verstehbar werden.

Der Artikel verortet sich innerhalb der medical geography in der geography of health care (Philo, 2009:451), speziell im Bereich der hospital spaces. In jüngerer Zeit ist - aufbauend auf Arbeiten von Massey (2005) und Thrift (2008) - eine konstruktivistischere Lesart der Bedeutung von place in der Organisation von health care zentraler geworden (Street und Coleman, 2012:10).

Frühgeborenen-Intensivstation werden ihrerseits v.a. aus epidemiologischer, medizinischer, ethischer, aber auch soziologischer Perspektive untersucht (Orfali, 2004:2009). ${ }^{3}$ Während sich aus sozialwissenschaftlicher Sicht ein großer Teil der Literatur in diesem Teilbereich mit der rationalethischen Entscheidungsfindung (z.B. Orfali, 2004; Vermeulen, 2004) oder aber dem Beziehungsaufbau unter den Bedingungen der Ungewissheit des (Weiter-)Lebens (z.B. Navne et al., 2017; Widding und Farooqi, 2016) auseinandersetzt, wird die Konstitution der Frühgeborenen-Intensivstation v.a. vonseiten der Science und Technology Studies (STS) thematisiert; hier ist besonders Mesman sehr aktiv (u.a. 2008, 2009, 2012).

Street und Coleman (2012:10 f.) kritisieren an Mesmans Perspektive die Konzeption der FrühgeborenenIntensivstation als isolierte Inselhaftigkeit und plädieren für eine stärker kontextualisierende Sichtweise, die hospital spaces gleichzeitig als einerseits von der restlichen Gesellschaft abgegrenzt und andererseits relational mit ihr verbunden konzipiert. Außerdem tendiere die STS dazu, die Rolle von Emotionen zu vernachlässigen. Während Street und Coleman auf Foucaults Konzept der Heterotopie zurückgreifen, um die ,,komplexe alltägliche Konfiguration und Anordnung von Materialitäten, sozialen Praktiken und Repräsen-

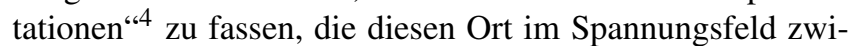
schen Stabilität und Wandel konstituieren (2012:5 f.), nutzt der vorliegende Artikel eine andere theoretische Grundlage: die Praxistheorie des US-amerikanischen Sozialtheoretikers Theodore Schatzki. Diese Theorie basiert auf materiellen Arrangements und sozialen Praktiken als zentralen Kon-

\footnotetext{
${ }^{3}$ Aus sozialwissenschaftlicher Perspektive gibt es im Vergleich zum medizinisch-epidemiologischen Blickwinkel extrem wenig Literatur zu diesem Themenfeld. Hinzu kommt, dass sich der Ort der Frühgeborenen-Intensivstation drastisch gewandelt hat, sodass ältere Literatur einen Ort mit anderen Praktiken und Arrangements beschreibt und daher für die in diesem Aufsatz verfolgte Zielsetzung nicht hilfreich ist.

${ }^{4}$ Hier und im Folgenden eigene Übersetzung.
}

zepten. Praktiken und Arrangements bilden komplex miteinander verwobene „Bündel“", die konstitutiv für Orte sind, aber gleichzeitig relational über räumlich begrenzte Orte hinausweisen. Diese theoretische Konzeption verspricht daher einen Mehrwert bei der geographischen Untersuchung der Konstitution einer Frühgeborenen-Intensivstation, wie in der Folge gezeigt wird. ${ }^{5}$

Die Praxistheorie Schatzkis hat in den letzten Jahren in den Sozialwissenschaften insgesamt (für einen Überblick: Schäfer, 2016) sowie speziell in der Geographie zunehmend Beachtung gefunden (für einen Überblick: Everts et al., 2011, seitdem u. a. Dünckmann und Fladvad, 2016; LahrKurten, 2012; Maus, 2015 sowie die Beiträge in diesem Themenheft).

Erkenntnistheoretisch ist der vorliegende Beitrag damit in einer breiten konstruktivistischen Strömung verortet, genauer gesagt in einem an Bedeutung gewinnenden Paradigma, das Performativität und Materialität in den Mittelpunkt rückt und „Sozialität diesseits von Kognition, Rationalität oder Normativität" analysiert (Gugutzer, 2015:117). ${ }^{6}$ Der practice turn wird ,,von einem ganzen Bündel durchaus heterogener sozialwissenschaftlicher Ansätze verfolgt" (Schmidt, 2012:11).

Es wird deutlich werden, dass Schatzkis Praxistheorie ein sehr gut geeignetes Instrumentarium darstellt, um den practice turn geographisch in Wert zu setzen. Zu diesem Zweck wird im Folgenden kurz in das Analysevokabular der Schatzki'schen Praxistheorie eingeführt, um im Anschluss diese Begrifflichkeit als Analysekategorien für die empirische Untersuchung einer Frühgeborenen-Intensivstation zu nutzen.

\section{Schatzkis Praxistheorie}

Im Zuge eines practice turn wird die Bedeutung von Performativität und Materialität deutlich aufgewertet (Schatzki et al., 2001; Schmidt, 2012; Gugutzer, 2015). Diesem Denken wird der US-amerikanische Sozialtheoretiker Theodore Schatzki mit seiner Praxistheorie gerecht: Sie berücksichtigt in der gegenwärtigen Diskussion intensiv thematisierte Konzeptualisierungen sozialer Phänomene wie etwa Emotionalität oder Materialität, ohne „klassische“ Konzepte komplett aufzugeben, diese aber auch nicht überzubetonen: „Intentionalität, Normativität und [symbolische] Schemata [werden] in ihrem Status jedoch grundsätzlich modifiziert, wenn

\footnotetext{
${ }^{5}$ Die Praxistheorie Schatzkis und ihr analytischer Mehrwert werden gegenwärtig stark diskutiert (eine lohnende aktuelle Lektüre, die vielfältige Perspektiven einbezieht und Schatzkis Ansatz auch mit anderen Ansätzen vergleicht, bietet der Sammelband von Hui et al., 2017).

${ }^{6}$ Dies bedeutet keineswegs ein komplettes Negieren „klassischer" handlungstheoretischer Elemente wie Intentionalität oder Normativität, sondern lediglich ein Hinterfragen ihres herausgehobenen Status in einem breiter angelegten sozialtheoretischen Vokabular (s. Kapitel 2).
} 
man davon ausgeht, dass Handeln im Rahmen von Praktiken [... ] begriffen werden kann“ (Reckwitz, 2003:291 f.). All diese Konzeptualisierungen sozialer Phänomene werden von Schatzki in die beiden übergeordneten Konzepte einbezogen: „Alle sozialen Phänomene sind Ausschnitte oder Aspekte eines riesigen, sich ständig transformierenden Netzes von Praktiken und Arrangements" (Schatzki, 2010:130). Soziale Praktiken und materielle Arrangements sind die beiden zentralen Konzepte, mit denen Schatzki sein Theoriegebäude errichtet.

Damit vereint Schatzki in seiner Theorie Elemente zweier Strömungen, die üblicherweise in getrennten Theorien ausgearbeitet werden: Praktiken-Theorien und ArrangementTheorien. ${ }^{7}$ Während Erstere sehr grob gesagt „organisierte Aktivitäten“ in den Blick nehmen, thematisieren Letztere, ebenfalls vereinfacht, ,,in Konfigurationen organisierte soziale Phänomene“" (Schatzki, 2002:xii f.). Schatzki beansprucht für sich, im Gegensatz zu anderen Theoretikern, die beiden Konzepte Praktiken und Arrangements explizit und detailliert auszuarbeiten und durch ihre Verknüpfung einen umfassenden Analyserahmen für die Untersuchung sozialer Phänomene anzubieten (Schatzki, 2011:8, 2012:14).

Schatzkis Theoriegebäude kann stark vereinfacht wie folgt dargestellt werden: Praktiken lassen menschliches Tun begreifbar werden, während Arrangements die Kontexte dieses Tuns beschreiben. Zentral ist, dass Praktiken und Arrangements zusammenhängen, sogenannte practice-arrangementBündel (practice-arrangement-bundle) bilden. Dies betont erstens den klassischen Gedanken der Rekursivität von Praktiken und Arrangements (sie ,ermöglichen und beschränken einander“ zugleich; Schatzki, 2002:117). ${ }^{8}$ Zweitens, und viel wichtiger, lässt die Betonung dieser Verwobenheit soziale Phänomene, wie in der Folge zu zeigen sein wird, erst in ihrer ganzen Komplexität abbildbar werden.

Eine soziale Praktik wird von Schatzki als „Aktivitäten,Bündel', d.h. [als] ein organisierter Zusammenhang körperlicher Taten und Äußerungen“ definiert (Schatzki, 2002:71). Die körperlichen Taten und Äußerungen bilden die kleinsten Bausteine von Praktiken (s. Abb. 1). Sie sind mittels vier verschiedener organisierender Elemente zu Praktiken verbunden: Erstens das „Praktische Verstehen“, das einerseits das Wissen umfasst, welche körperlichen Taten und Äußerungen eine Praktik konstituieren, sowie das Know-how, diese auszuführen. Andererseits beschreibt praktisches Verstehen aber auch die Fähigkeit, Praktiken zu erkennen, also die sozial grundlegende Intelligibilität. Zweitens das „Grundle-

\footnotetext{
${ }^{7} \mathrm{Zu}$ den Praktiken-Theorien zählt Schatzki u. a. die Arbeiten von Bourdieu, Giddens und Taylor; als Arrangement-Theorien fasst er die Theorien von Latour, Callon, Laclau/Mouffe, Deleuze/Guattari und Foucault zusammen (Schatzki, 2002:xii f.).

${ }^{8}$ Ich bezeichne den Gedanken der Rekursivität im Anschluss an Ortmann als klassisch: „Diesen Gedanken, so bedeutsam er ist, finden wir in der Sozialtheorie, ich bin geneigt zu sagen: schon immer vor"“ $(2008: 102)$
}

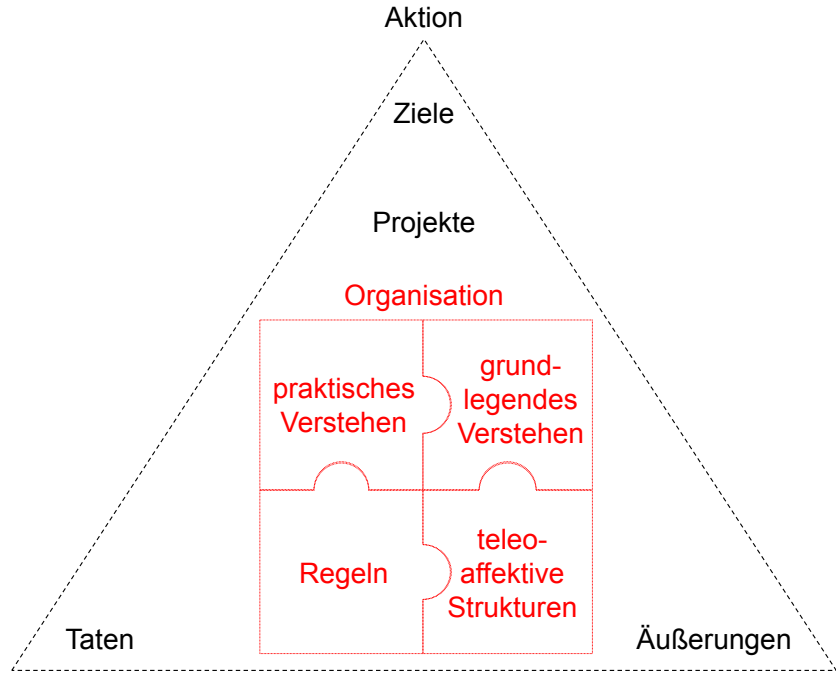

Abb. 1. Aktions- und Organisations-Komponenten einer Praktik. Quelle: eigener Entwurf nach Schatzki (2002).

gende Verstehen", das abstrakter ist und etwa Dinge wie religiöse Überzeugungen oder Gemeinschaftsgefühl umfasst. Drittens „Regeln“ als selbst- oder fremdauferlegte Vorgaben, d.h. selbstdefinierte Beschränkungen oder aber z.B. gesetzliche Regelungen. Viertens „Teleoaffektive Strukturen“, welche die Aktions- und Organisations-Komponente einer Praktik über den teleologischen Aspekt vorhandener Ziele verschränken, aber auch affektive Elemente, z.B. Emotionen, Stimmungen etc. beinhalten. Gerade im Element der teleoaffektiven Strukturen schlägt sich Schatzkis Einschätzung nieder, dass „Bourdieu (und zu einem geringeren Grad auch Giddens) Handlung zu stark als aus Kontrolle und Beherrschung ablaufend darstellt“ (Schatzki, 1997:301). Nur unter Berücksichtigung dieser Konzeption von Handeln ist der Begriff der Ziele im Rahmen von Praktiken zu verstehen.

Das zweite zentrale Konzept - die materiellen Arrangements - definiert Schatzki als „Ensembles von Entitäten, durch und inmitten derer das soziale Leben passiert die Arrangements von Menschen, Artefakten, Organismen und Dingen, die menschliche Koexistenz charakterisieren" (Schatzki, 2002:38). Schatzki priorisiert gegenüber anderen Ansätzen wie etwa der ANT die menschliche Handlungsfähigkeit in der Konstituierung des Sozialen (ebd.: $190 \mathrm{ff}$.). Dennoch berücksichtigt auch er die Beziehungen zwischen den genannten Entitäten, solange sie als sozial bedeutungsvoll gelten können. Als Resultat sind Arrangements als Anordnungen bedeutungsvoller materieller Entitäten anzusehen, die ihre Bedeutung im Rahmen von Praktiken erhalten. ${ }^{9}$

\footnotetext{
${ }^{9}$ Schatzki verweist an dieser Stelle darauf, dass die „Relationen, Positionen und Bedeutungen genau wie die Arrangements, deren Gesichtspunkte sie sind, labile Phänomene sind, von denen nur vorübergehende Fixierungen möglich sind“" (Schatzki, 2002:24). Da-
} 
Die bereits erwähnte Zusammenführung sozialer Praktiken und materieller Arrangements im Konzept der practicearrangement-Bündel ist für seine Theorie zentral: Laut Schatzki bilden Praktiken und Arrangements Bündel, indem (1) Praktiken materielle Arrangements hervorbringen, gebrauchen, verändern, auf sie gerichtet oder untrennbar mit ihnen verbunden sind und (2) Arrangements Praktiken ausrichten, präfigurieren und ermöglichen (Schatzki, 2016:33).

Das Soziale insgesamt besteht in der Praxistheorie Schatzkis folglich aus einem ,riesigen, sich ständig transformierenden Netz von Praktiken und Arrangements" (Schatzki, 2010:130). So wie sich mittels Intelligibilität einzelne Praktiken identifizieren lassen, sind innerhalb dieses Netzes des Sozialen einzelne „Konstellationen“ von practicearrangement-Bündeln voneinander unterscheidbar, da diese „dichter" verwoben sind (Schatzki, 2012:17). Solch eine Konstellation ist z.B. eine Universität, die Warenkette von Espresso oder eben die Frühgeborenen-Intensivversorgung in Deutschland. Diese Unterscheidungen sind bis zu einem gewissen Grad kontingent, Abgrenzungen werden nicht immer gleich getroffen.

In Schatzkis Praxistheorie bilden Praktiken und Arrangements somit eine „emergente Ebene des Sozialen“, die von einer flachen Ontologie geprägt ist $-\mathrm{d}$. h. es gibt keine Strukturen jenseits bzw. oberhalb von Praktiken, Arrangements und den Bündeln, die sie bilden. ${ }^{10}$ Die practicearrangement-Bündel stellen für Schatzki den Ort des Sozialen bzw. die ,sites of the social" dar, wobei der Begriff der „Site“ hierbei nicht mit „Standort“ zu übersetzen ist. Dennoch sind practice-arrangement-Bündel als komplex miteinander verwobene Praktiken und materielle Arrangements geographisch höchst bedeutend: Sie sind konstitutiv für Orte, ein practice-arrangement-Bündel ist allerdings nicht deckungsgleich mit einem Ort. Vielmehr weisen immer Relationen über einen Ort hinaus und verbinden das gesamte Netz des Sozialen. ${ }^{11}$ Dies wird bei der empirischen Untersuchung einer Frühgeborenen-Intensivstation zu beachten sein, um method(olog)isch eine ,präsentistische“" Forschung (Niewöhner et al., 2012:41) zu vermeiden, die lediglich das vor Ort Beforschte berücksichtigt.

Das Ziel dieses Aufsatzes, den Ort der FrühgeborenenIntensivstation verstehbar werden zu lassen, lässt sich mit

mit ist Schatzkis Praxistheorie zumindest poststrukturalistisch anschlussfähig (vgl. Moebius, 2008).

${ }^{10}$ Schatzkis Praxistheorie hat einen in mehrfacher Hinsicht überbrückenden Charakter: Sie dezentriert durch die Fokussierung auf Praktiken und Arrangements das Subjekt und vermeidet damit auch eine Dichotomisierung von Körper und Geist sowie von Subjekt und Objekt; gleichzeitig greift die Praxistheorie weder auf autonome Subjekte noch auf vorgängige Strukturen zurück, sodass es ihr gelingt, eine Brücke zwischen Individualismus und Holismus zu schlagen.

${ }^{11}$ Eine detailliertere theoretische In-Bezug-Setzung von Schatzkis Praxistheorie (die er selbst als ,Site Ontology“ bezeichnet) und Raumkonzepten wäre durchaus wünschenswert. dem nun erarbeiteten theoretischen Vorwissen in folgender Frage konkretisieren: Welche sozialen Praktiken und materiellen Arrangements konstituieren den Ort FrühgeborenenIntensivstation?

Schatzkis Praxistheorie liefert hierzu das AnalyseVokabular: In der Folge wird mithilfe dieses Vokabulars eine dichte Beschreibung im Sinne Geertz' einer konkreten Frühgeborenen-Intensivstation vorgenommen, um die Konstitution dieses Ortes zu verstehen. Es wird deutlich werden, dass die dichte Beschreibung Erkenntnisse liefert, ohne auf eine (erklärende) „,Intellektualisierung “ des Sozialen“ zurückgreifen zu müssen (Reckwitz, 2003:296). Die Praxistheorie beansprucht, dadurch dem Sozialen näherzukommen.

Die empirische Grundlage dieser Analyse stellt ein fünfmonatiger Aufenthalt des Autors auf einer FrühgeborenenIntensivstation dar. Hintergrund war die Frühgeburt seines Sohnes, sodass es sich um eine Art von Autoethnographie handelt, die gerade im Bereich der geography of health care des Öfteren eingesetzt wird (z.B. Anderson, 2006; Richards, 2008). Der Autor strebte hierbei eine analytische Autoethnographie an, die versucht, vom subjektiven Einzelfall zu verallgemeinern - wohlwissend, dass eine vollständige „Objektivierung“ des Erlebten nicht möglich ist. Neben dieser teilnehmenden Beobachtung wurden ExpertinnenInterviews geführt (längere Gespräche mit 12 Krankenschwestern, 4 Ärztinnen, 8 Eltern und einer Physiotherapeutin) sowie Dokumenten- und Literaturanalyse vorgenommen.

\section{Intensive Geographien - Drei Frühchen}

In den OECD-Staaten ist eine flächendeckende Versorgung auch für sehr kleine Frühgeborene gegeben. Dennoch existieren große Unterschiede, v.a. in der Organisation dieser flächendeckenden Versorgung, in der absoluten und relativen Anzahl von Frühgeburten sowie der Überlebenswahr-

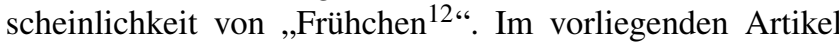
wird die dichte Beschreibung einer konkreten deutschen Frühgeborenen-Intensivstation angestrebt.

Um die Konstitution der Frühgeborenen-Intensivstation verstehbar zu machen, wird die dichte Beschreibung mittels drei Frühchen erzählt: Es handelt sich um ein und denselben Körper, der jedoch in drei unterschiedliche practicearrangement-Bündel einbezogen ist: Medizin, Familie und Gesundheitsökonomie, die insgesamt die wesentlichen Elemente der Frühgeborenen-Intensivversorgung umfassen.

Damit wird eine konzeptionelle Anleihe bei Mol (2002) vorgenommen, die den „multiplen“ Körper untersuchte. Das erkenntnistheoretische und zugleich dramaturgisch strukturierende Potential dieser Multiplizität eignet sich sehr gut für das Verstehbarmachen der Frühgeborenen-Intensivstation aus praxistheoretischer Perspektive: Laut Mol (2002:4 f.) werden Objekte durch Praktiken hergestellt, weswegen diese Praktiken in den Vordergrund gerückt werden müssen. In

\footnotetext{
${ }^{12}$ Frühchen wird als Synonym für Frühgeborene verwendet.
} 
jeder dieser Praktiken ,ist [das Objekt] etwas anderes“ (Law und Mol, 2008:59), die multiplen Objekte bilden jedoch $\mathrm{Zu}$ sammenhänge (Mol, 2002:5).

Unterschiedliche Praktiken inszenieren (enact) damit das Objekt jeweils neu. Diese enactments sind zugleich sozial und materiell (Michael und Rosengarten, 2012:3), weswegen sich die Schatzki'sche Praxistheorie mit ihren zentralen Konzepten der sozialen Praktiken und der materiellen Arrangements hervorragend für die Nutzung der Mol'schen Multiplizität eignet. ${ }^{13}$ Mit dem epistemologischen Schwenk der Multiplizierung wird zugleich die betrachtete Realität ,dichter".

Das multiple Frühchen wird folglich in drei abgrenzbaren practice-arrangement-Bündeln konstituiert; jedes dieser drei Frühchen wird zu einem unterschiedlichen Zeitpunkt ,geboren“: Das medizinische Frühchen kommt im Moment der leiblichen Geburt auf die Welt - zumeist wird es per Kaiserschnitt geboren und benötigt unmittelbar danach eine Erstversorgung, die die lebensnotwendigen Funktionen sicherstellt (nicht selten mittels Apparaturen z.B. zur Beatmung; Jorch, 2006:25 ff.). Das familiäre Frühchen wird in dem Moment geboren, in dem zumeist der Vater trotz der allzu oft schwierigen Umstände die ersten Glückwünsche erhält. Das gesundheitsökonomische Frühchen kommt schließlich im Moment der Aufnahme in die Krankenversicherung zur Welt, die für die erbrachten Leistungen aufkommt.

In der Folge werden die einzelnen Frühchen und die sie konstituierenden relational miteinander verbundenen practice-arrangement-Bündel dargestellt. Das InBeziehung-Setzen dieser practice-arrangement-Bündel führt $\mathrm{zu}$ interessanten Reibungspunkten, die neue Einsichten gewähren und daher als wichtiges Element ebenfalls dargelegt werden.

\subsection{Medizinisches Frühchen}

Das medizinische Frühchen wird von einem practicearrangement-Bündel konstituiert, das vor allem die Sicherstellung der Lebensfunktionen des Frühgeborenen zum Ziel hat: Ein extremes Frühchen ist gewöhnlich noch nicht in der Lage, selbst zu atmen, seine Körpertemperatur zu halten oder zu trinken. Daher müssen diese Funktionen mithilfe zumeist technischer Apparaturen gewährleistet werden, sodass das Arrangement eines Frühgeborenen-Stellplatzes neben dem Inkubator (für Körpertemperatur und Luftfeuchtigkeit) auch noch zahlreiche weitere Geräte umfassen kann, z.B. Beatmungsgeräte, Perfusoren-Ständer mit einer bzw. mehreren Spritzenpumpen (sog. ,Perfusoren“ zur exakt dosierten intravenösen Verabreichung von Medikamenten), Absaugvorrich-

\footnotetext{
${ }^{13}$ Annemarie Mols Konzept der „Multiplizität“ ist im heterogenen Feld der Akteur-Netzwerk-Theorie (ANT) verortet. Ihr Konzept lässt sich innerhalb Schatzkis Praxistheorie auch deshalb nutzen, da Schatzki seine Theorie in kritischer Diskussion mit den „Arrangement-Theorien“ (u.a. ANT) entwirft und deswegen konzeptionelle Ähnlichkeiten bestehen.
}

tungen (etwa zum Entfernen von Lungensekret) oder Sauerstoffschläuche (für eine schnelle Sauerstoffzufuhr, falls der Körper des Frühchens nicht ausreichend mit Sauerstoff gesättigt ist). Durch die Vielzahl an Gerätschaften und deren zahlreiche Kabel und Schläuche wird nicht nur die Optik des Stellplatzes stark geprägt. Vielmehr reichen die Zugänge der Gerätschaften bis in den Körper des Frühchens, sodass zwischen dem Körper und den Apparaturen nicht wirklich getrennt werden kann.

Ist dieses Netzwerk von Gerätschaften, in dessen Zentrum der Körper des Frühgeborenen liegt, erst einmal konstituiert, muss es im Rahmen von Praktiken der FrühgeborenenÜberwachung kontrolliert werden. Mittels eines Monitors und weiterer Kabel werden die lebensnotwendigen Körperfunktionen des Frühchens von Krankenschwestern rund um die Uhr überwacht: Herzfrequenz, Atemfrequenz und Sauerstoffsättigung werden jeweils Ober- und Untergrenzen zugewiesen, bei deren Über- oder Unterschreiten ein Alarm ertönt. ${ }^{14}$ Hierbei wird noch zwischen einem gelben Alarm und einem roten Alarm unterschieden. Während gelbe Alarme des Öfteren von Bewegungen des Frühgeborenen ausgelöste Fehlalarme bedeuten oder auch einfach eine kurze Weile abgewartet werden kann, ist bei roten Alarmen oft schnelles Eingreifen nötig, um die wesentlichen Körperfunktionen wieder in den Bereich innerhalb der definierten Grenzwerte zu bringen. Neben den Monitoralarmen besitzen nahezu alle Gerätschaften - Inkubatoren, Perfusoren, Beatmungsgeräte, Wärmebetten, Sauerstoffzufuhren usw. - ihre eigenen, akustisch je unterschiedlichen Alarme. Daher ist die Atmosphäre der Intensivstation stark geprägt von den diversen akustischen Signalen, die vor allem die Eltern sehr schnell in einen permanenten emotionalen Ausnahmezustand versetzen.

Die untersuchte Frühgeborenen-Intensivstation besitzt ein materielles Arrangement, das die FrühgeborenenÜberwachung erleichtert: Im zentralen Foyer der Station ist eine Theke derart positioniert, dass an diesem zentralen Arbeitsplatz sitzende Krankenschwestern einerseits zwei Monitore im Blick haben, auf denen die wichtigsten Werte aller Monitore der 18 Frühgeborenen-Stellplätze zusammengeführt werden; andererseits können von diesem Platz aus alle fünf Zimmer eingesehen werden, in denen die Frühchen liegen. Eines dieser Zimmer besitzt mehr Stellplätze als die anderen Zimmer; hier liegen die kleinsten Frühchen, sodass sich allein durch die erhöhte Anzahl an Frühgeborenen und die Intensität der Versorgung nahezu immer eine Krankenschwester in diesem Zimmer befindet, was die Frühgeborenen-Überwachung in diesem Raum noch einmal intensiviert. Auf der Rückseite der Theke sind die anderen Zimmer angeordnet (Arztzimmer, Besprechungszimmer, Küche, Umkleide, Labor, Stillzimmer, Vorratsraum).

Neben den Praktiken und Arrangements zur ersten Sicherstellung der Lebensfunktionen sowie jenen zu ihrer Überwa-

\footnotetext{
${ }^{14}$ Weitere überwachbare Funktionen sind möglich, werden aber zumeist nur in akuten postoperativen Phasen genutzt.
} 
chung gibt es noch solche, die das hergestellte Netzwerk und letztlich das Frühchen in dessen Zentrum - vor lebensbedrohlichen Krankheitserregern schützen sollen. Hierbei handelt es sich um Praktiken und Arrangements der Hygiene und des Desinfizierens, in deren Rahmen grundlegende räumliche Kammerungen der Frühgeborenen-Intensivstation stattfinden.

Die Praktiken der Hygiene und des Desinfizierens beginnen bereits vor Betreten der Station: In einem vorgelagerten Bereich gibt es ein Waschbecken, einen Desinfektionsspender sowie eine Anleitung zum richtigen Durchführen des Hände-Desinfizierens. Erst nach Durchführen dieser Praktik wird Zugang zur Station gewährt. Außerdem sind mitgeführte Gegenstände in Schließfächern außerhalb der Station zu deponieren. Nach Ankunft auf der Station müssen im Foyer Kittel über die Alltagskleidung angezogen werden. An der Außenwand der einzelnen Behandlungszimmer ist jeweils ein weiterer Desinfektionsspender angebracht, der vor Betreten des Zimmers zu benutzen ist. Die Behandlungszimmer selbst sind wiederum gekammert: Wird der Bereich eines Frühgeborenen-Stellplatzes verlassen, sind erneut die Hände zu desinfizieren.

Jenseits dieser horizontalen Kammerung existiert auch eine vertikale Zonierung der Hygiene: Den obersten Bereich auf dem Inkubator dürfen nur Ärztinnen und Krankenschwestern benutzen, eine Ablage an der Wand wird von den Krankenschwestern und den Eltern genutzt. Unterhalb dieser Ablage befindet sich der mittlere Bereich des Stellplatzes, in dem medizinisches Material (Sauerstoffhandpumpe, EinmalKanülen zum Absaugen von Lungensekret, Einmal-Spritzen zur Fütterung via Magensonde sowie der Desinfektionsspender, aber auch Windeln und Feuchttuicher) und technische Gerätschaften (Unterdruckgerät, Sauerstoffzufuhr) vorzufinden sind; dieser wird von Versorgungsschwestern gereinigt und aufgefüllt. Der unterste Bereich ist der Boden, der nicht steril ist und von Reinigungskräften gesäubert wird. Fällt etwas zu Boden, wird es entsorgt, es sei denn, es handelt sich um etwas „Wertvolles“. Letzteres (Kleidung, Spieluhr, Schmuck) muss vor einer erneuten Nutzung desinfiziert werden. Die vertikale Zonierung spiegelt folglich die Hierarchie des Personals einer Frühgeborenen-Intensivstation wider.

Erkrankt trotz dieser Vorkehrungen ein Frühchen an einem ansteckenden Erreger, ändern sich die Praktiken und Arrangements der Hygiene und des Desinfizierens, um den Krankheitserreger von den anderen Frühgeborenen fernzuhalten: Das infizierte Kind wird in einem der Behandlungszimmer isoliert. Außerdem müssen nun je nach Krankheitserreger Einmalkittel und evtl. Handschuhe getragen werden, sodass diese ebenso vor dem Zimmer zur Verfügung gestellt werden müssen wie auch ein Müllbehälter zu ihrer Entsorgung nach dem Verlassen des Zimmers. Die Reinigungs-Praktiken verändern sich, indem z.B. dieses Zimmer als letztes gereinigt wird. Erlaubt es die Belegungszahl an Frühchen, kann es vorkommen, dass eine Schwester sich ausschließlich um dieses Kind kümmert, um ihm eine intensivere Pflege zukommen zu lassen und die Gefahr der Verbreitung des Krankheitserregers zu reduzieren.

Insgesamt ist der physische Körper des Frühgeborenen folglich in ein Geflecht von Praktiken und Arrangements eingewoben: Zu diesen gehört die Konstitution eines Netzwerks zur Sicherstellung der grundlegenden Lebensfunktionen, in dessen Mittelpunkt sich der Körper des Babys befindet. Dieses Netzwerk wird im Rahmen der FrühgeborenenÜberwachung stabilisiert. Schließlich wurden Praktiken und Arrangements der Desinfektion und der Hygiene identifiziert, die eine horizontale Kammerung sowie eine vertikale Zonierung der Frühgeborenen-Intensivstation bewirken. All diese Praktiken und Arrangements bilden zusammen das practice-arrangement-Bündel, innerhalb dessen der Körper des Frühgeborenen als medizinisches Frühchen konstituiert wird.

\subsection{Familiäres Frühchen}

In einem praxistheoretischen Verständnis ist eine Familie nicht als ,natürlich vorkommende Ansammlung von Individuen“ zu verstehen. Vielmehr wird ,ihre Realität durch tägliche Aktivitäten wie das gemeinsame Essen konstruiert" (DeVault, 1991:39). Welche Praktiken sind also im Kontext einer Frühgeborenen-Intensivstation identifizierbar, mittels derer Familie konstruiert wird?

Familie wird zunächst durch erste, den Umständen entsprechende Glückwünsche (verbunden mit medizinischen Informationen) konstituiert, indem man/frau im Sinne eines Sprechakts als Vater/Mutter eines Sohns/einer Tochter adressiert und diskursiv konstituiert wird. Sprechakte lassen sich praxistheoretisch als Teil von Praktiken (Äußerungen) rahmen. Die Anrufungs-Äußerungen werden im Rahmen der weiteren Kommunikation auf der Station im Kontext unterschiedlicher Praktiken reproduziert (und gelegentlich modifiziert, wie später gezeigt wird).

Das familiäre Frühchen wird in diesem Verständnis also nach dem medizinischen Frühchen ,geboren“. Bevor auf der Frühgeborenen-Intensivstation weitere Praktiken die Familie konstituieren, muss das Frühchen jedoch nach der Erstversorgung zunächst auf die Frühgeborenen-Intensivstation transportiert und dort sein Stellplatz eingerichtet werden. Danach besucht zumeist erst nur der Vater das Baby, da die Mutter nach dem extremen Frühgeburten zumeist vorangehenden Kaiserschnitt nicht bzw. nur für sehr kurze Zeit dazu in der Lage ist. In den Tagen bzw. Wochen danach kommt es typischerweise zu einer Umkehrung dieser Verhältnisse, sodass tagsüber v.a. die Mütter auf der FrühgeborenenIntensivstation anzutreffen sind, während die Väter aufgrund ihrer Berufstätigkeit v.a. abends auf die Station kommen.

Die besonderen Umstände der Geburt als Frühchen führen wie bereits gesehen zu einem deutlich anderen Arrangement als bei einer normalen Geburt. Dieses bringt mit der beschriebenen Apparate-Umgebung und all seinen Alarmen sowie der Ungewissheit über die Zukunft des eigenen Kindes 
nicht zuletzt auch andere Emotionen mit sich und erschwert u.a. die Bindung zwischen den Eltern und dem Kind. ${ }^{15}$ Eine Mutter, deren zweites Kind eine Frühgeburt ist, beschreibt diese Situation folgendermaßen:

Es hat schon ein bisschen gedauert, bis da richtige Muttergefühle aufkamen. Das war nicht so, wie ich das kenne. Dass man entbindet und das Kind anguckt und überquillt vor Liebe. Das war sehr viel Angst und Traurigkeit. (Christ, 2014:15-25)

Auf einer Frühgeborenen-Intensivstation wird die Bindung zwischen Eltern und Frühchen - und damit die Familie - v.a. durch die zumeist nach einigen Tagen einsetzenden Praktiken und Arrangements des sogenannten „Kangarooing“ sowie des „Begrenzens“ etabliert. Sie sind wichtige Möglichkeiten des Körperkontakts, da andere babytypische Versorgungspraktiken wie Füttern, Wickeln und Baden durch die Eltern bei extremen Frühchen zumeist erst nach einigen Wochen einsetzen können.

Speziell das Kangarooing ist für die Eltern eine Möglichkeit, ,zu ,kuscheln', d.h. ihrem Kind durch Körperkontakt Zärtlichkeit zu geben“ (Jorch, 2006:99): Das Frühchen wird beim Kangarooing aus dem Inkubator herausgenommen und der Mutter/dem Vater auf die unbekleidete Brust gelegt. Vater oder Mutter befinden sich hierzu auf einem Stuhl mit verstellbarer Rückenlehne, sodass eine auch über längere Zeit bequeme, nahezu liegende Position eingenommen werden kann. Das Kangarooing kann einige Stunden dauern, sollte jedoch nicht zu kurz währen, damit die positiven Aspekte des Körperkontakts den Stress der Umgebungsveränderung überwiegen. Diese Praktik gehört für die Eltern zu den emotional schönsten Momenten während ihrer Zeit auf der Intensivstation und ist - soweit es die (akustischen) Umstände zulassen - geprägt von Ruhe und Zufriedenheit, was sehr häufig auch dem Gesichtsausdruck der Eltern während des Kangarooing anzusehen ist.

Ist das Frühgeborene nicht stabil genug, um aus dem Inkubator herausgenommen $\mathrm{zu}$ werden, oder steht $\mathrm{zu}$ wenig Zeit zur Verfügung, kann der Körperkontakt zwischen Eltern und Kind auch über das sogenannte Begrenzen hergestellt werden. Hierbei begrenzt der Vater/die Mutter mit beiden Händen das Frühchen an Kopf und Füßen bzw. an Kopf und Rumpf.

Mittlerweile sind die familiären Praktiken des Kangarooing und des Begrenzens auch von medizinischer Seite anerkannt, da beide durch den Körperkontakt positive Auswirkungen auf die Entwicklung des Frühgeborenen haben. Dies war jedoch nicht immer so, vielmehr haben sich viele Praktiken und Arrangements von Frühgeborenen-Intensivstationen deutlich verändert. Bis etwa 1980 war Eltern sogar der Zutritt zu Frühgeborenen-Intensivstationen als potentielle Gefah-

\footnotetext{
${ }^{15}$ Vor allem in einem Unterbereich der medical anthropology wird dieser Aspekt untersucht (z.B. Navne et al., 2017; Widding und Farooqi, 2016)
}

renquelle nicht gestattet (Marcovich und de Jong, 2003:82). Viele ältere Stationen besitzen daher noch Balkone, von denen aus die Eltern in die Station schauen durften, um ihr Kind zu sehen.

Zusammenfassend lässt sich sagen, dass die Familie durch Anrufungs-Äußerungen konstituiert und immer wieder reproduziert wird. Darüber hinaus wird Familie v.a. durch Praktiken und Arrangements konstruiert, in denen mit dem Fokus auf zärtlichen körperlichen Kontakt gemeinsam Zeit verbracht wird.

\section{In-Beziehung-Setzen des medizinischen und des familiären Frühchens}

Es wird schnell deutlich, dass sich die beiden practicearrangement-Bündel, die das medizinische und das familiäre Frühchen konstituieren, nicht strikt trennen lassen: So verbringen auch Krankenschwestern im Rahmen medizinischer Praktiken viel Zeit mit den Frühchen und haben viel Körperkontakt mit diesen. Außerdem bringen sie den Babys in der Regel viel Zärtlichkeit entgegen, die sich laut Aussage mehrerer Krankenschwestern aus dem Beweggrund, diesen Beruf gewählt zu haben, speist.

Krankenschwestern sind zudem auch selbst in familiären Arrangements und Praktiken engagiert und hätten nicht selten einen bisher unerfüllten Kinderwunsch, sodass Frühchen zu „Ersatzkindern“ werden könnten, wie eine Schwester erklärt. Eine andere Krankenschwester beschreibt einer Kollegin ein Baby mit den Worten: „Als ich ihn das erste Mal auf dem Arm hatte, habe ich gedacht: ,Den gebe ich nicht mehr her!' “.

Durch das gemeinsame Engagement in Praktiken, in denen Körperkontakt und Zärtlichkeit geteilt werden, entstehen folglich auch zwischen Krankenschwestern und Frühchen intensive soziale Beziehungen (eine Schwester spricht bei der Entlassung eines Frühchens von „Trennungsschmerz"). Diese äußern sich auch in der Zuteilung der Versorgung der Frühgeborenen durch Schwestern im Rahmen der Praktik der Verteilung der Babys zu Beginn einer Schicht. Eine Krankenschwester erklärt einer Kollegin die Wahl eines Frühchens: „Die ist so süß mit Kulleraugen - da habe ich sie mir heute gleich wieder geholt". Die sich derart im Lauf der Zeit vertiefenden sozialen Beziehungen befinden sich in Konkurrenz zu den sich entwickelnden Eltern-Kind-Beziehungen: Einerseits freuen sich die Eltern, dass ihr Kind zärtlich von den Krankenschwestern behandelt wird, andererseits ist Zärtlichkeit ihre Domäne. Wenn Krankenschwestern das betreute Frühchen beispielsweise mit „mein Hase“ anreden, kann das Possessivpronomen Gefühle der Eifersucht hervorrufen, da diese Anrufungs-Äußerung in Konflikt mit den etablierten familiären Anrufungs-Äußerungen steht (z.B. „Ihr Sohn“ vs. „mein Hase“).

Das Gefühl von Eifersucht kann noch dadurch verstärkt werden, dass auf einer Frühgeborenen-Intensivstation zur Sicherstellung des Überlebens der Frühchen medizinische 
Praktiken Vorrang vor familiären Praktiken haben. So kommt es gelegentlich zu Situationen, in denen der die Körperfunktionen des Frühgeborenen überwachende Monitor einen roten Alarm auslöst, weil ein Grenzwert unterschritten wird: Die Schwester eilt zu Hilfe, schiebt die das Frühchen begrenzende Mutter zur Seite und sagt dann zum Kind: „Mein Spatz, was machst Du denn?“".

Situationen wie die beschriebene führen bei den Eltern auch zu Gefühlen von Ohnmacht. Eltern fühlen sich durch die niedrigere Bedeutung der familiären Praktiken, in denen sie sich engagieren, gegenüber den medizinischen Praktiken auch sehr oft wie Praktikantinnen der Krankenschwestern. Dies äußert sich am stärksten, wenn die Krankenschwestern beginnen, die Eltern in Praktiken der Versorgung (Wickeln, Baden, Füttern per Magensonde etc.) einzuweisen. Die Einweisung ist nötig, da die Unreife des Frühchens sowie die Praktiken und Arrangements der Intensivstation sich von den häuslichen unterscheiden. Die so entstandenen Rollen können trotz der sich entwickelnden Erfahrung erstaunlich stabil bleiben, sodass Eltern auch nach Monaten auf Station von einigen Krankenschwestern noch wie Praktikantinnen behandelt werden können - dieser Aspekt scheint zusätzlich gegendert, indem Vätern zumeist weniger Erfahrung zugeschrieben wird als Müttern.

Insgesamt existieren neben den naheliegenden Gefühlen von Angst und Traurigkeit, aber auch der wachsenden Liebe der Eltern viele weitere Emotionen, ohne die sich der Ort Frühgeborenen-Intensivstation nicht verstehen lässt: Vonseiten der Eltern können dies einerseits Eifersucht und Ohnmacht dem pflegenden Personal gegenüber sein; andererseits sind Krankenschwestern häufig auch Bezugspersonen, die Verständnis für die Situation, die Ängste und Sorgen der Eltern haben und sich mit ihnen über erreichte Entwicklungsschritte freuen können.

\subsection{Gesundheitsökonomisches Frühchen}

Die Versorgung eines Frühgeborenen ist mit Aufwand verbunden, der im Kontext eines marktwirtschaftlichen Systems als Kosten in Rechnung gestellt wird. Diese Kosten werden in der Bundesrepublik Deutschland in aller Regel von den Krankenversicherungen übernommen.

Bis zum Jahr 2005 war jedes Krankenhaus in Deutschland berechtigt, extrem Frühgeborene intensivmedizinisch $\mathrm{zu}$ versorgen. Etwa jedes fünfte Krankenhaus hielt eine Frühgeborenen-Intensivversorgung vor; insbesondere in städtischen Regionen wurde dieses Angebot zur Anwerbung Schwangerer eingesetzt (Gerber et al., 2008:1439). Seither hat sich die Frühgeborenen-Intensivversorgung stark verändert - vor allem durch die Ökonomisierung des Gesundheitssystems im Rahmen der Gesundheitsreform der Agenda 2010, welche die makroökonomische Wettbewerbsfähigkeit Deutschlands durch eine Senkung der Lohnnebenkosten verbessern sollte (Deutscher Bundestag, 2003:71).
In einem ersten Schritt wurde im Jahr 2006 ein ,,neonatologisches Versorgungskonzept“" eingeführt. Dieses etablierte eine dreistufige Hierarchie der Frühgeborenen-Versorgung: Extreme Frühgeburten dürfen nur noch in „Perinatalzentren Level 1" versorgt werden, starke Frühgeburten benötigen mindestens „Perinatalzentren Level 2“, späte Frühchen ,perinatale Schwerpunkte“ (Gemeinsamer Bundesausschuss, 2005:2). Damit wurde eine „Risikozentralisation“ vorgenommen (MDS, 2009:10), deren Ziel eine Optimierung der Versorgung nach dem Risikoprofil bei gleichzeitiger Sicherung der flächendeckenden Versorgung ist (Gemeinsamer Bundesausschuss, 2005:2) - faktisch bedeutet die Optimierung den Erhalt der Versorgungsqualität bei Senkung der Kosten. Seither muss im Rahmen von Akkreditierungs-Praktiken die Erfüllung von infrastrukturellen sowie personellen Anforderungen nachgewiesen werden; einige Krankenhäuser boten in der Folge keine Frühgeborenen-Intensivversorgung mehr an.

Eine zentrale Rolle in der Reformierung der Frühgeborenen-Intensivversorgung in Deutschland spielt die „Mindestmengendebatte“ (MDS, 2009:10), bei der es sich um eine „kontroverse Debatte“ (Gerber et al., 2008:1439) handelt; hierbei steht die Frage im Mittelpunkt, ob die Versorgungsqualität durch die Einführung einer Mindestfallzahl verbessert werden kann. Es existieren verhärtete Fronten zwischen den diese Regelung befürwortenden Medizinischen Fachgesellschaften und den Gesundheitsversicherern einerseits sowie den ablehnenden Haltungen der Deutschen Krankenhausgesellschaft und der Bundesärztekammer andererseits. Während Erstere mit einer besseren und weniger kostenintensiven Versorgung argumentieren (Zimmer, 2012: 517), sehen Letztere die rein quantitative Argumentation einer Mindestmenge als Verkürzung der Problematik der Versorgungsqualität an, die u.a. die Anstrengungen, Frühgeburten überhaupt erst zu vermeiden, ausblende.

Im Zuge dieser Debatte wurden im Rahmen analysierender Praktiken Studien und Gutachten erstellt (z.B. Gerber et al., 2008; MDS, 2009), die u.a. die FrühgeborenenIntensivversorgung anderer Staaten betrachteten. Es kommt allerdings zu keiner „einheitlichen Interpretation“ der Ergebnisse dieser Studien. Minimaler Konsens war die „Einführung einer (nicht evidenzbasierten) Regelmäßigkeitszahl zum Ausschluss von Gelegenheitsversorgung“ im Jahr 2008 (MDS, 2009:10), die aus juristischen Gründen zum Jahr 2010 in eine geringe Mindestmengenregelung von 14 Frühgeborenen pro Jahr für die Perinatalzentren Level 1 sowie Level 2 (also für extreme und starke Frühchen) überführt wurde. Dadurch sank die Zahl der Krankenhäuser, die in Deutschland extreme Frühgeburten versorgen dürfen, weiter. ${ }^{16}$ Für das Jahr 2011 war eine weitere Anhebung der Mindestmengen-

\footnotetext{
${ }^{16}$ Eine genaue Zahl existiert nicht. Die Zahl der Perinatalzentren Level 1 und Level 2 wurde 2013 jedoch auf ca. 150-200 Krankenhäuser geschätzt, was weniger als zehn Prozent der existierenden Krankenhäuser bedeutet (GKinD, 2013).
} 
regelung auf 30 Frühgeburten geplant, die nach einer Klage von ca. 40 kleineren Krankenhäusern allerdings außer Vollzug gesetzt wurde (Gemeinsamer Bundesausschuss, 2010).

Dennoch scheint die Mehrheit der gesundheitsökonomischen Akteure weiterhin mit einer Erhöhung der Mindestmengenregelung zu rechnen. So raten etwa Falb und Magunia von Roland Berger Strategy Consultants: „Die Verschärfung der [Mindestmengen-]Regelung ist nur noch eine Frage der Zeit. Eine große Anzahl der [Stationen] wird in $\mathrm{Zu}$ kunft gezwungen sein, entweder über die kritische Mindestmengenschwelle zu wachsen oder [...] mit dem Verlust eines attraktiven Erlössegments zu rechnen. Die Entwicklung einer [...] Expansions- oder Rückzugsstrategie ist erforderlich“ (Falb und Magunia, 2011:3).

Das gesundheitsökonomische Frühchen stellt ein ,attraktives Erlössegment“ dar, denn die intensivmedizinische „Versorgung von Frühgeborenen wird gut vergütet": Ein Frühchen mit einem Geburtsgewicht von $550 \mathrm{~g}$ und keinen Komplikationen benötigt ungefähr vier Monate stationärer Behandlung, was mit etwa 100000 Euro entgolten wird (Zimmer, 2012:517). Daher finden einige kleinere Krankenhäuser ihre „Wachstumsstrategie“ in der Bildung von Verbünden durch innovative Praktiken und Arrangements (v.a. systematische Personalrotation und standortübergreifende Videokonferenzen mit dem Ziel einer ,lernenden Organisation“; Hochbaum, 2011:49).

Die attraktive Vergütung der FrühgeborenenIntensivversorgung ist vor dem Hintergrund eines zunehmend ökonomisierten Krankenhaussektors zu bewerten, in dem ,die wirtschaftlichen Schwierigkeiten insbesondere in kommunalen Krankenhäusern zunehmend bedrohlicher werden und teilweise bis hin zur Insolvenzgefährdung reichen“ (Deloitte Consulting, 2013:3). Genau dies ist jedoch erklärtes Ziel der Gesundheitsreform, die eine „Intensivierung des Preiswettbewerbs" in einem nun etablierten „Wachstumsmarkt“" erzeugen will, indem „,verfestigte marktferne Strukturen“ zugunsten einer „wettbewerblichen Orientierung" aufgebrochen werden sollen (Deutscher Bundestag, 2003:72 ff.). Erreicht wurde dies v.a. dadurch, dass bis 2010 schrittweise die "Erlöse regulatorisch begrenzt“ wurden (per Fallkostenpauschalsystem), während ,auf der Kostenseite weitestgehend marktwirtschaftliche Dynamiken" herrschen; gleichzeitig haben auch die Bundesländer ihre Krankenhausfinanzierung drastisch reduziert. Daraus ergibt sich eine „Deckungslücke“ (Deloitte Consulting, 2013: 3), die den Krankenhaussektor bereits deutlich verändert hat: Von 2002 bis 2013 kam es bei einer rückläufigen Gesamtzahl an Krankenhäusern $(-10 \%)$ zu einem massiven Verlust kommunal und freigemeinnützig geführter Kliniken ( $-27 \%$ bzw. $-19 \%$ ), während die Zahl privater Krankenhäuser deutlich zugenommen hat $(+32 \%$; Deloitte Consulting, 2013, Statistisches Bundesamt, 2015). ${ }^{17}$

\footnotetext{
${ }^{17}$ Auf die äußerst spannende Frage, inwiefern aufgrund der Ersetzung der gemeinwohlorientierten Krankenhäuser durch die gewin-
}

Für zahlreiche Krankenhäuser sind folglich die Einnahmen aus der Frühgeborenen-Intensivversorgung wesentliche Finanzierungsquellen. Aufgrund der Ökonomisierung des Gesundheitssystems haben in allen Kliniken - in privat geführten wie kommunalen - betriebswirtschaftliche Praktiken stark an Bedeutung gewonnen. Dies führt dazu, dass ein „großes Risiko“ besteht, dass Mediziner „weniger medizinisch und moralisch völlig unabhängige Entscheidungen treffen" (Zimmer, 2012:518). So könnte im Bereich der Versorgung extremer Frühgeburten , der Anreiz bestehen, die eine oder andere Geburt in die Wege zu leiten oder einzelne Kinder nicht $\mathrm{zu}$ verlegen, um eine Mindestmenge zu überschreiten" (Gerber und Rossi, 2010:362).

Insgesamt wird das gesundheitsökonomische Frühchen folglich von einem practice-arrangement-Bündel konstituiert, das in Deutschland durch die Gesundheitsreform gegenwärtig einen grundlegenden Wandel erfährt: Mit der Einführung des neonatologischen Versorgungskonzepts dürfen unterschiedliche Arten von Perinatalzentren verschiedene Grade unreifer Frühchen behandeln. Diese Spezialisierung wurde spürbar verstärkt durch die Einführung der Mindestmengenregelung und der damit sinkenden Zahl an Krankenhäusern, die extreme Frühgeburten versorgen.

\section{In-Beziehung-Setzen des gesundheitsökonomischen und des medizinischen Frühchens}

Die betrachtete Frühgeborenen-Intensivstation besitzt den höchsten Level (Perinatalzentrum Level 1), kann also die extremsten Frühchen medizinisch versorgen. Parallel zu der im Zuge der Reformen tendenziell intensivierten medizinischen Versorgung auf diesen Stationen gibt es gerade im Frühgeborenen-Bereich große medizinische Fortschritte. Die Auswirkungen für die auf extreme Frühchen spezialisierten Stationen beschreibt eine Krankenschwester wie folgt:

So gut der medizinische Fortschritt auch ist, führt er dazu, dass immer mehr Kinder immer früher geholt werden - das sind dann auch Fast-Aborte so in der 22. Woche. Das ist anstrengend, weil immer mehr Apparate nötig sind.

Dadurch, dass die Medizin immer unreifere Frühchen versorgen kann, stellt sich nicht zuletzt auch die Frage nach der Grenze der Lebensfähigkeit immer wieder neu. Diese Frage ist eine gesamtgesellschaftliche Frage und wird von ökonomischen, medizinischen, ethischen und weltanschaulichen Parametern aufgespannt und in Deutschland anders beantwortet als z.B. in Frankreich, den Niederlanden oder der Schweiz: In Deutschland existiert ein Graubereich in der 24. Schwangerschaftswoche; davor wird von Maßnahmen der

norientierten Kliniken die flächendeckende und wohnortnahe Daseinsvorsorge gewährleistet ist, kann an dieser Stelle nicht näher eingegangen werden. Bereits die Ausgangslage, ob am „Krankenhausmarkt" eine „Überversorgung“ besteht, wird kontrovers diskutiert (Deloitte Consulting, 2013:3). 
„Lebensverlängerung“ eher abgeraten, danach ist „,im Regelfall eine lebenserhaltende Therapie anzustreben" (AWMF, 2014:6).

Durch die Spezialisierung und den medizinischen Fortschritt ändern sich folglich die die Station konstituierenden Praktiken und Arrangements der FrühgeborenenIntensivversorgung. Extremere Frühchen sind noch unreifer, sodass eine größere Zahl kleinster Frühgeborener auch dazu führt, dass eher medizinische Praktiken überwiegen; demgegenüber sind es jedoch oft gerade die babytypischen Praktiken des Fütterns, Badens, Wickelns, die mit positiven Emotionen besetzt sind, nun jedoch immer seltener ausgeübt werden können. Eine Krankenschwester berichtet Folgendes:

Wir haben momentan 18 Kinder, nur beatmete. Keins, das man mal füttern könnte [mit dem Fläschchen]. Dann sterben natürlich auch viele. Naja, ... ist stressig.

Die Intensivst-Medizin ist nicht nur für Eltern, sondern auch für das Personal emotional sehr anstrengend. Die aufgebauten sozialen Beziehungen führen u.a. dazu, dass Krankenschwestern auch zu einigen Beerdigungen gehen. Daher verweisen viele Krankenschwestern darauf, dass man auf einer Frühgeborenen-Intensivstation nicht sein ganzes Leben arbeiten könne - eine wahrgenommen hohe Fluktuation ist die Folge. Die betrachtete Station versuchte jedoch gerade mit der Erfahrung des Personals die Versorgungsqualität zu steigern, wie eine Ärztin erklärt:

Eine erfahrene Schwester findet immer noch einen Zugang [für eine Infusion o.ä. in der Peripherie, d.h. den Extremitäten des Körpers], während auf Stationen mit weniger erfahrenem Personal schneller ein Zentralkatheter gelegt wird, was wieder ein Infektionsrisiko bedeutet.

Erfahrung wird auf der untersuchten Station folglich als ein Faktor angesehen, der in der Suche nach Balance zwischen „medizinischen und gesundheitsökonomischen“ Aspekten (MDS, 2009:20) zu berücksichtigen ist. Daher wird auf dieser Station versucht, erfahrene Krankenschwestern und Ärztinnen zu halten.

\section{Fazit}

Der Aufsatz nahm den Ort der FrühgeborenenIntensivstation in den Blick, der in der öffentlichen Diskussion nahezu abstinent ist. Ziel war es, diesen Ort verstehbar zu machen. Hierzu wurde unter Rückgriff auf das Analysevokabular der Praxistheorie Schatzkis der Ansatz einer dichten Beschreibung verfolgt.

Dabei wurden analytisch drei Frühchen unterschieden, die jeweils durch ein practice-arrangement-Bündel konstituiert sind - ein medizinisches, ein familiäres und ein gesundheitsökonomisches Frühchen: Das medizinische Früh- chen wird durch Praktiken und Arrangements konstruiert, die das Netzwerk zur Sicherstellung der grundlegenden Körperfunktionen herstellen und überwachen sowie gefährdende Krankheitserreger fernhalten sollen. Das familiäre Frühchen konstituiert sich durch Anrufungs-Äußerungen sowie mittels Praktiken und Arrangements, in denen in gemeinsam verbrachter Zeit Körperkontakt und Zärtlichkeit im Mittelpunkt stehen. Das gesundheitsökonomische Frühchen wird durch Praktiken und Arrangements konstituiert, welche das Gesundheitssystem produzieren, reformieren und (verändert) reproduzieren, wie etwa die Akkreditierung sowie die unterschiedliche Spezialisierung der einzelnen Frühgeborenen-Intensivstationen. Der Wandel des räumlich ausgedehnteren gesundheitsökonomischen practice-arrangement-Bündels verändert auch die konkreten lokalen Frühgeborenen-Intensivstationen: Vor dem Hintergrund des medizinischen Fortschritts werden auf der betrachteten Station durch diese gesundheitsökonomischen Veränderungen in zunehmenden Maße intensivmedizinische Praktiken und Arrangements notwendig. Die Ökonomisierung des Gesundheitssystems hat folglich ganz konkrete Auswirkungen auf die medizinischen Praktiken und Arrangements einzelner Frühgeborenen-Intensivstationen - wobei dies einerseits eine quantitative und qualitative Intensivierung der Versorgung bedeuten, andererseits aber auch die Schließung von Stationen nach sich ziehen kann.

Aus geographischer Perspektive ist wesentlich, dass der soziale Ort der Frühgeborenen-Intensivstation durch diese drei grundlegenden und relational miteinander verbundenen practice-arrangement-Bündel konstituiert wird. Erstens wird dadurch der konstruierte Charakter von Ort und Raum offensichtlich: Wie die ihn konstituierenden Elemente - soziale Praktiken und materielle Arrangements in ihrer Verwobenheit - besitzt auch der soziale Ort niemals eine Stabilität, sondern wird immer wieder aufs Neue produziert. Zweitens wird der relationale Charakter der Konstitution von Ort deutlich und betont die Bedeutung einer Sensibilität des Forschenden für die über den physischen Ort hinausweisenden Relationen: Der Ort der Frühgeborenen-Intensivstation ist nur in seiner relationalen Einbettung in ein über den konkreten lokalen Kontext hinausgehendes System verstehbar. Hiermit kann auch die Forderung von Street und Coleman eingelöst werden, hospital spaces gleichzeitig als abgegrenzt und zutiefst mit der Gesellschaft verbunden zu denken (2012:5).

Sehr wichtig ist hierbei, dass die relationale Einbettung keiner Logik einer Mikro-Makro-Dichotomie folgt, in der zwei ontologisch differente Ebenen unterschieden werden. Diese Perspektive einer „flachen Ontologie“ vertritt nicht zuletzt die Praxistheorie Schatzkis (vgl. Schatzki, 2016) - die praxistheoretische Perspektive liegt „quer zu dieser epistemologischen Bifurkation" in Mikro und Makro (Schmidt, 2012:235). Schatzki spricht daher von „groß“ und „klein“, von räumlich mehr oder weniger weit ausgedehnten practicearrangement-Bündeln, wobei die Beziehungen zwischen diesen beiden ,,vielfältiger, kontingenter und viel weniger syste- 
matisch sind, als es die theoretische Position anerkennt, die von abgrenzbaren Ebenen ausgeht" (Schatzki, 2016: 39).

Folglich lässt sich die Praxistheorie nicht auf eine inhärente Mikrologik reduzieren, der zufolge die Gegenstände praxistheoretischer Untersuchungen Mikrophänomene sein müssten, die wiederum lediglich Ausdruck einer übergeordneten Makrostruktur seien. Praxistheorien eignen sich zur Untersuchung „,großer“ wie „kleiner“ Phänomene - jenseits einer überholten Mikro-Makro-Dichotomie. Alle sozialen Phänomene sind somit auf einer einzigen ontologischen Ebene verortet. Damit konzipiert die Praxistheorie auch Gesellschaft nicht als „Makrophänomen“, sondern als „,iterative Hervorbringung der sozialen Wirklichkeit in einem fortlaufenden, sich immer wieder aufs Neue in ähnlicher, regelmäBiger Weise vollziehenden sprachlichen, körperlichen, materiellen und symbolischen Geschehen - das heißt in und durch soziale Praktiken" (Schmidt, 2012:14).

Bei der dichten Beschreibung einer FrühgeborenenIntensivstation wurde deutlich, dass Emotionalität eine sehr wichtige Rolle spielt: Neben den offensichtlichen Emotionen Angst, Traurigkeit, Hoffnung kommen nun auch die positiven Emotionen entstehender enger Bindungen zwischen Krankenschwestern und Frühchen ebenso in den Blick wie Machtverhältnisse nicht nur zwischen Schwestern und Ärztinnen, sondern auch von Krankenschwestern und Eltern; letztere können zu Situationen führen, in denen bei den Eltern die Gefühle von Eifersucht und Ohnmacht vorherrschen. Oft vernachlässigt werden auch die Emotionen des Personals einer Frühgeborenen-Intensivstation, die aus der Spezialisierung und dem technischen Fortschritt resultieren, da positiv besetzte babytypische Versorgungspraktiken zugunsten intensivmedizinischer Praktiken zurückgedrängt werden. Der hierdurch erzeugte Stress kann eine erhöhte Fluktuation des Personals auf diesen Stationen mit sich bringen, welche die angestrebten Effekte der Spezialisierung ad absurdum führen könnte (im Rahmen der Debatte um die Mindestmengenregelung wird implizit gerade mit dem Erfahrungswert des Personals argumentiert, der über eine Mindestfallzahl quantifiziert werden soll).

Insgesamt wurde deutlich, dass Körperlichkeit, Materialität und Emotionalität einen wesentlichen Teil der Sozialität einer Frühgeborenen-Intensivstation darstellen. Für diese Aspekte des Sozialen sensibilisieren gerade die Ansätze des practice turn, da sie eben diese sozialen Elemente fokussieren. Hierbei besitzt das praxistheoretische Vokabular Schatzkis gegenüber anderen Ansätzen von practice und material turn v.a. zwei Stärken: Erstens arbeitet Schatzki die beiden Konzepte von Praktiken und Arrangements sowie ihre Verwobenheit im Rahmen der practice-arrangement-Bündel detailliert aus. Das Konzept der Praktiken wird als Handeln im Spannungsfeld routinisierter Geschlossenheit und performativer Offenheit konzipiert und berücksichtigt auch gleichberechtigt affektives, nicht-rationales Handeln. Diese Konzipierung stellt die zweite Stärke der Praxistheorie Schatzkis dar - die Integration von Emotionalität in das zentrale
Konzept der Praktiken, während andere materialistische Sozialtheorien Emotionalität nicht ausreichend berücksichtigen (u.a. Street und Coleman, 2012:10). ${ }^{18}$

Nichtsdestotrotz lassen sich zwei Forschungsdesiderate erkennen: Erstens sollte angesichts der aktuellen Debatte um Emotionalität und Affekt die Aktualität der Konzeption von Emotionalität in der Schatzki'schen Praxistheorie kritisch geprüft werden ${ }^{19}$; dies verspricht zweitens eine gewinnbringende Perspektive bei der Diskussion, wie die Rolle von Emotionalität bei der Konstitution von Orten und Räumen ontologisch gefasst werden kann - die FrühgeborenenIntensivstation ist hierbei lediglich ein ,frappierendes“ (uncanny) Beispiel, dass diese Rolle oft sehr wichtig sein kann auch bei sogenannten „banaleren“ Emotionen und Orten als es im vorliegenden Artikel thematisiert wurde.

Datenverfüg barkeit. Für diesen Artikel wurden keine Datensätze genutzt.

Interessenkonflikt. Der Autor erklärt, dass kein Interessenkonflikt besteht.

Danksagung. Ich danke Florian Weisser, den beiden anonymen Gutachter*innen sowie Jonathan Everts und Tim Elrick als Herausgebern des Themenhefts für ihre konstruktiven Hinweise und Anregungen.

Edited by: Benedikt Korf

Reviewed by: two anonymous referees

\section{Literatur}

Anderson, L.: Analytic Autoethnography, J. Contemp. Ethnogr., 35, 373-395, 2006.

Arbeitsgemeinschaft der Wissenschaftlichen Fachgesellschaften (AWMF): Gemeinsame Empfehlung: Frühgeborene an der Grenze der Lebensfähigkeit, https: //www.aem-online.de/fileadmin/user_upload/Publikationen/ S2k_Fruehgeburt_Grenze_Lebensfaehigkeit_2014-09_1_.pdf (letzter Zugriff: 4 November 2018), 2014.

Bäurle, A.: Weltfrühgeborenentag, https://www.aerztezeitung. de/medizin/krankheiten/infektionskrankheiten/article/947694/ weltfruehgeborenentag-anzahl-fruehchen-sieht-deutschland-alt. html (letzter Zugriff: 17 Februar 2018), 2017.

\footnotetext{
${ }^{18}$ So schreiben Street und Coleman (2012:10): „Moreover, the STS focus on expert systems und materiality can lead to a neglect of the role of imagination und emotion in the constitution of hospital space".

${ }^{19}$ In der Geographie sind in dieser Diskussion v.a. Smith et al. (2009) sowie Pile (2010) zu nennen. Die große epistemologische Nähe zu Schatzki macht z.B. den Ansatz von Wetherell (2012) hierfür besonders interessant.
} 
Blencowe, H., Cousens, S., Chou, D., Oestergaard, M., Say, L., Moller, A.-B., Kinney, M., and Lawn, J.: Born Too Soon: The global epidemiology of 15 million preterm births, Reprod Health, 10(Suppl 1): S2, 1-14, 2013.

Christ, A.: Eine Handvoll Leben, Extreme Frühgeburten und ihre Chancen, Dokumentarfilm ausgestrahlt am 12. September 2014 auf arte, 2014.

Deloitte Consulting: Strategiestudie 2013, Herausforderungen für kommunale Krankenhäuser in Deutschland, Berlin, 2013.

Deutscher Bundestag: Gesetzentwurf der Fraktionen SPD, CDU/CSU und BÜNDNIS 90/DIE GRÜNEN, Entwurf eines Gesetzes zur Modernisierung der gesetzlichen Krankenversicherung (GKV-Modernisierungsgesetz - GMG), Drucksache 15/1525, Berlin, 2003.

DeVault, M. L.: Feeding the Family, The Social Organization of Caring as Gendered Work, The University of Chicago Press, Chicago, 1991.

Dünckmann, F. und Fladvad, B.: The Practice of Changing the Rules of Practice: An Agonistic View on Food Sovereignty, Geogr. Z., 104, 25-49, 2016.

Everts, J., Lahr-Kurten, M., and Watson, M.: Practice Matters! Geographical inquiry und theories of practice, Erdkunde, 65, 323334, 2011.

Falb, R. und Magunia, P.: Perinatalzentren im Wandel. Status und Handlungsbedarf in der Perinatalmedizin in Deutschland, http://www.rolandberger.de/media/pdf/Roland_Berger_ Perinatalzentren_im_Wandel_20110323.pdf (letzter Zugriff: 5 Mai 2016), 2011.

Gemeinsamer Bundesausschuss: Vereinbarung des Gemeinsamen Bundesausschusses über Maßnahmen zur Qualitätssicherung der Versorgung von Früh- und Neugeborenen, BAnz, S. 15.684, 2005.

Gemeinsamer Bundesausschuss: Bekanntmachung eines Beschlusses des Gemeinsamen Bundesausschusses über eine befristete Außervollzugesetzung einer Änderung der Mindestmengenvereinbarung: Mindestmengen für Früh- und Neugeborene Perinatalzentren Level 1, BAnz, S. 4.480, 2010.

Gerber, A. und Rossi, R.: Neonatologische Versorgung - Fallzahlregelung. Einfluss auf Qualität und Finanzierung pädiatrischer Einrichtungen, Monatsschr. Kinderh., 158, 356-363, 2010.

Gerber, A., Lauterbach, K., und Lüngen, M.: Perinatalzentren. Manchmal ist weniger mehr, Dtsch. Arztebl. A, 105, 1439-1441, 2008.

Gesellschaft der Kinderkrankenhäuser und Kinderabteilungen in Deutschland (GKinD): G-BA-Beschluss zur Richtlinie für die Versorgung von Früh- und Neugeborenen vom 20.06.2013, https://www.gnpi.de/cms2/images/attachments/static/Brief_ BMG_Rau_2013_11_05_sig.pdf (letzter Zugriff: 4 November 2018), 2013.

Gugutzer, R.: Soziologie des Körpers, 5. Aufl., Transcript, Bielefeld, 2015.

Hochbaum, K.: Qualität als Zukunftsfaktor, KU Gesundheitsmanagement, 12, 48-50, 2011.

Hui, A., Schatzki, T., and Shove, E. (Hrsg.): The Nexus of Practices. Connections, constellations, practitioners, Routledge, 2017.

Jorch, G.: Frühgeborene, Urania, Stuttgart, 2006.

Kirschner, W., Halle, H., und Pogonke, M.-A.: Kosten der Frühund Nichtfrühgeburten und die Effektivität und Effizienz von
Präventionsprogrammen am Beispiel von BabyCare, Präv. Gesundheitsf., 4, 41-50, 2009.

Lahr-Kurten, M.: Deutsch sprechen in Frankreich, Praktiken der Förderung der deutschen Sprache im französischen Bildungssystem, transcript, Bielefeld, 2012.

Law, J. und Mol, A.: The Actor-Enacted: Cumbrian Sheep in 2001, in: Material Agency: Towards a Non-Anthropocentric Approach, Herausgeber: Knappett, C. und Malafouris, L., Springer, Boston, 57-77, 2008.

Marcovich, M. und de Jong, T.: Frühgeborene - zu klein zum Leben?, 4. Aufl., Fischer, Frankfurt/Main, 2003.

Massey, D.: For Space, Sage, London, 2005.

Maus, G.: Erinnerungslandschaften: Praktiken ortsbezogenen Erinnerns am Beispiel des Kalten Krieges, Kieler Geographische Schriften Band 127, Kiel, 2015.

Medizinischer Dienst des Spitzenverbandes Bund der Krankenkassen e.V. (MDS): Umsetzung der Vereinbarung über Maßnahmen zur Qualitätssicherung der Versorgung von Früh- und Neugeborenen, Essen, 2009.

Michael, M. und Rosengarten, M.: Medicine: Experimentation, Politics, Emergent Bodies, Body Soc., 18, 1-17, 2012.

Moebius, S.: Handlung und Praxis. Konturen einer poststrukturalistischen Praxistheorie, in: Poststrukturalistische Sozialwissenschaften, Herausgeber: Moebius, S. and Reckwitz, A., Suhrkamp, Frankfurt am Main, 58-74, 2008.

Mol, A.: the body multiple, ontology in medical practice, Duke University Press, Durham, 2002.

Navne, L., Svendsen, M., and Gammeltoft, T.: The Attachment Imperative: Parental Experiences of Relation-making in a Danish Neonatal Intensive Care Unit, Med. Anthropol. Q., 32, 120-137, https://doi.org/10.1111/maq.12412, 2017.

Niewöhner, J., Sørensen, E., and Beck, S.: Einleitung, Science und Technology Studies - Wissenschafts- und Technikforschung aus sozial- und kulturanthropologischer Perspektive, in: Science und Technology Studies, Herausgeber: Beck, S., Niewöhner, J., and Sørensen, E., Eine sozialanthropologische Einführung, Transcript, Bielefeld, 9-48, 2012.

Orfali, K.: Parental role in medical decision-making: fact or fiction? A comparative study of ethical dilemmas in French und American neonatal intensive care units, Soc. Sci. Med., 58, 2009-2022, 2004.

Ortmann, G.: Organisation und Welterschließung. Dekonstruktionen, 2. Aufl., VS Verlag für Sozialwissenschaften, Wiesbaden, 2008.

Philo, C.: Medical geography, in: Gregory, D., Johnston, R., Pratt, G., Watts, M., and Whatmore, S. (Hrsg.): Dictionary of Human Geography, 5. Aufl., Wiley-Blackwell, Chichester, 451453, 2009.

Pile, S.: Emotions und affect in recent human geography, T. I. Brit. Geogr., 35, 5-20, 2010.

Reckwitz, A.: Grundelemente einer Theorie sozialer Praktiken: Eine sozialtheoretische Perspektive, Z. Soziol., 32, 282-301, 2003.

Richards, R.: Writing the Othered Self: Autoethnography und the Problem of Objectification in Writing About Illness und Disability, Qual. Health Res., 18, 1717-1728, 2008.

Schäfer, H.: Einleitung: Grundlagen, Rezeption und Forschungsperspektiven der Praxistheorie, in: Praxistheorie, Ein Forschungsprogramm, Herausgeber: Schäfer, H., Transcript, Bielefeld, 925,2016 
Schatzki, T. R.: Practices und Actions. A Wittgensteinian Critique of Bourdieu und Giddens, Philos. Soc. Sci., 27, 283-308, 1997.

Schatzki, T. R.: The site of the social: a philosophical account of the constitution of social life und change, Pennsylvania State University Press, University Park, 2002.

Schatzki, T. R.: Materiality und Social Life, Nat. Cult., 5, 123-149, 2010.

Schatzki, T. R.: Where the Action Is (On Large Social Phenomena Such as Sociotechnical Regimes), Working Paper, http: //www.sprg.ac.uk/uploads/schatzki-wp1.pdf (letzter Zugriff: 5 Mai 2016), 2011.

Schatzki, T. R.: A primer on practices. Theory und research, in: Practice-Based Education, Herausgeber: Higgs, J., Barnett, R., Billett, S., Hutchings, M., und Trede, F., Perspectives und Strategies, Sense, Rotterdam, 13-26, 2012.

Schatzki, T. R.: Practice Theory as Flat Ontology, in: Praxistheorie, Ein Forschungsprogramm, Heausgeber: Schäfer, H., Transcript, Bielefeld, 29-44, 2016.

Schatzki, T. R., Knorr Cetina, K., and v. Savigny, E. (Hrsg.): The Practice Turn in Contemporary Theory, Routledge, London, 2001.
Schmidt, R.: Soziologie der Praktiken, Konzeptionelle Studien und empirische Analysen, Suhrkamp, Berlin, 2012.

Smith, M., Davidson, J., Cameron, L., and Bondi, L.: Geography und emotion - emerging constellations, in: Emotion, Place und Culture, Herausgeber: Smith, M., Davidson, J., Cameron, L., and Bondi, L., Ashgate, Farnham, 1-20, 2009.

Statistisches Bundesamt: Eckdaten der Krankenhäuser 2013, https://www.destatis.de/DE/ZahlenFakten/ GesellschaftStaat/Gesundheit/Krankenhaeuser/Tabellen/ KrankenhaeuserJahreVeraenderung.html (letzter Zugriff: 22 Mai 2016), 2015.

Street, A. und Coleman, S.: Introduction: Real und Imagined Spaces, Space Cult., 15, 4-17, 2012.

Thrift, N.: Non-representational theory: space, politics, affect, Routledge, Abingdon, 2008.

Vermeulen, E.: Dealing with doubt: Making decisions in a neonatal ward in The Netherlands, Soc. Sci. Med., 58, 2071-2085, 2004.

Wetherell, M.: Affect und Emotion, A New Social Science Understanding, Sage, London, 2012.

Widding, U. und Farooqi, A.: I thought he was ugly: Mothers of extremely premature children narrate their experiences as troubled subjects, FEM Psychol., 26, 153-169, 2016.

Zimmer, K.-P.: Neonatology Departments Under Economic Pressure, Dtsch. Arztebl. Int., 109, 517-518, 2012. 